\title{
Familial Atypical Hemolytic Uremic Syndrome: A Review of Its Genetic and Clinical Aspects
}

\author{
Fengxiao Bu, ${ }^{1,2}$ Nicolo Borsa, ${ }^{3,4}$ Ardissino Gianluigi, ${ }^{4}$ and Richard J. H. Smith ${ }^{1,2}$ \\ ${ }^{1}$ Interdepartmental PhD Program in Genetics, University of Iowa, Iowa City, IA52242, USA \\ ${ }^{2}$ Division of Nephrology, Department of Internal Medicine, Carver College of Medicine, University of Iowa, 5270 CBRB, \\ Iowa City, IA 52242, USA \\ ${ }^{3}$ Laboratory of Molecular Genetics, Fondazione IRCCS Cà Granda Ospedale Maggiore Policlinico, Milan 20122, Italy \\ ${ }^{4}$ Center for HUS Control, Fondazione IRCCS Cà Granda Ospedale Maggiore Policlinico, Milan 20122, Italy
}

Correspondence should be addressed to Richard J. H. Smith, richard-smith@uiowa.edu

Received 6 July 2012; Accepted 24 September 2012

Academic Editor: Michael A. Flierl

Copyright (C 2012 Fengxiao Bu et al. This is an open access article distributed under the Creative Commons Attribution License, which permits unrestricted use, distribution, and reproduction in any medium, provided the original work is properly cited.

\begin{abstract}
Atypical hemolytic uremic syndrome (aHUS) is a rare renal disease (two per one million in the USA) characterized by microangiopathic hemolytic anemia, thrombocytopenia, and acute renal failure. Both sporadic ( $80 \%$ of cases) and familial (20\% of cases) forms are recognized. The study of familial aHUS has implicated genetic variation in multiple genes in the complement system in disease pathogenesis, helping to define the mechanism whereby complement dysregulation at the cell surface level leads to both sporadic and familial disease. This understanding has culminated in the use of Eculizumab as first-line therapy in disease treatment, significantly changing the care and prognosis of affected patients. However, even with this bright outlook, major challenges remain to understand the complexity of aHUS at the genetic level. It is possible that a more detailed picture of aHUS can be translated to an improved understanding of disease penetrance, which is highly variable, and response to therapy, both in the short and long terms.
\end{abstract}

\section{Introduction}

Hemolytic uremic syndrome (HUS) is a rare disease characterized by microangiopathic hemolytic anemia, thrombocytopenia, and acute renal failure. It is most frequently caused by infections of Shiga-like toxin producing bacteria, such as Escherichia coli strain O157:H7, O111: $\mathrm{H} 8, \mathrm{O} 103: \mathrm{H} 2$, O123, and $\mathrm{O} 26$ [1]. In approximately 10\% of HUS cases, there is no association with Shiga-like toxin. These cases are classified as atypical HUS (aHUS) and occur with an incidence of about 2 per million in the USA [1,2]. aHUS patients have a poorer prognosis than those with typical HUS, with acute phase aHUS mortality of about $8 \%[3,4]$, and with $50 \%-80 \%$ of aHUS patients progressing to endstage renal failure [1]. However, it is important to note that epidemiological outcomes data are relatively out of date because of the development of better diagnostic, treatment, and management strategies.

Atypical HUS can be classified as sporadic or familial. Familial aHUS is defined as the presence of aHUS in at least two members of the same family with diagnoses at least 6 months apart $[1,3,5]$. It accounts for less than $20 \%$ of aHUS cases [3]. In familial aHUS (and also sporadic aHUS), genetic (e.g., gene mutations, rare variants, and risk haplotypes) and acquired abnormalities (e.g., autoantibodies against factor $\mathrm{H}$ ) are found in $\sim 70 \%$ of patients [6]. Gene mutations are usually found in complement genes, such as factor $\mathrm{H}(C F H)$, factor I $(C F I)$, factor B $(C F B)$, complement component $3(C 3)$, and membrane cofactor protein $(M C P$ or $C D 46)$. Evidence from familial studies indicates a high rate of incomplete penetrance, with about $50 \%$ of carriers of $C F H$ or $M C P$ aHUS-associated variants not developing disease [7]. The reasons underlying incomplete penetrance are unclear, although it is recognized that multiple predisposing genetic variants and risk haplotypes exist which may be relevant to disease onset in the face of environmental triggers such as pregnancy, viral infection, cancer, organ transplantation, and the use of certain drugs $[8,9]$.

In this paper, we focus on familial aHUS. Over the past 20 years, dozens of aHUS pedigrees have been reported, clarifying the underlying mechanisms of both familial and sporadic aHUS. In followed sections, we will discuss 


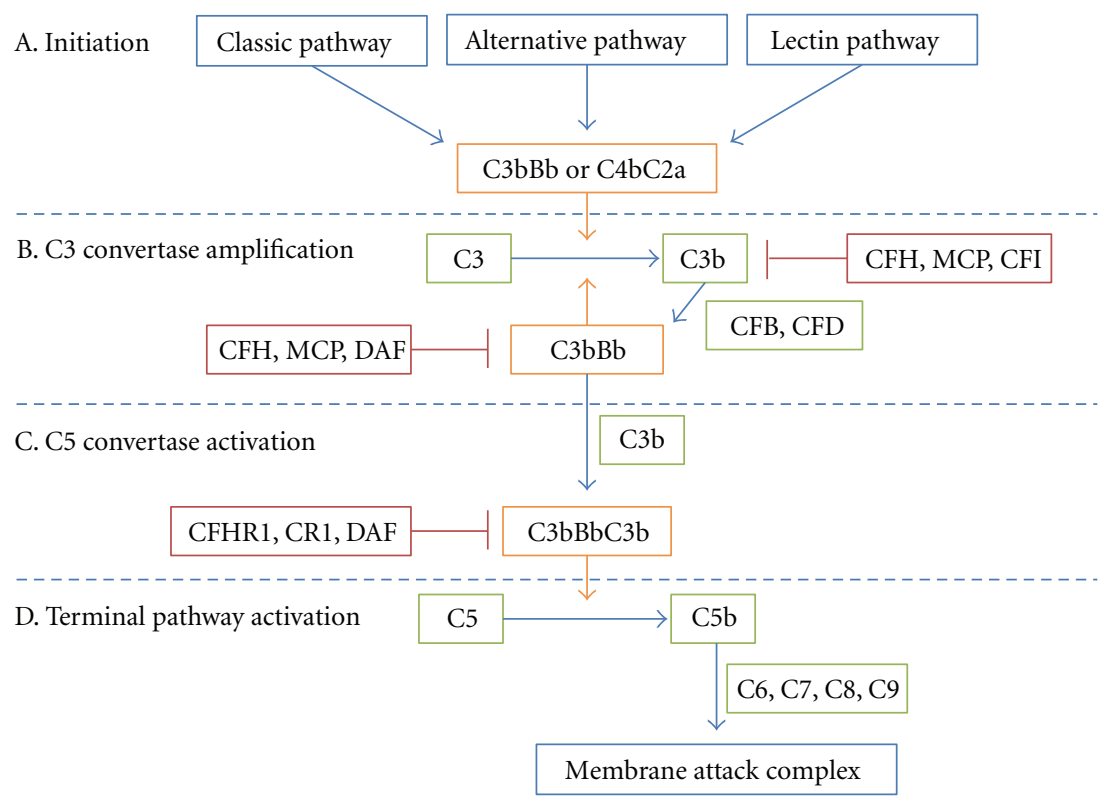

FIgURE 1: The complement system and its regulators. The complement system has four main steps. (A) Classical, alternative, or lectin pathway activation produces $\mathrm{C} 3$ convertases $(\mathrm{C} 3 \mathrm{bBb}$ or $\mathrm{C} 4 \mathrm{bC} 2 \mathrm{a})$ to initiate the complement cascade. (B) $\mathrm{C} 3$ convertase cleaves $\mathrm{C} 3$ into $\mathrm{C} 3 \mathrm{a}$ and $\mathrm{C} 3 \mathrm{~b}$. CFB binds to $\mathrm{C} 3 \mathrm{~b}$ and is cleaved by CFD into Bb, forming a new $\mathrm{C} 3$ convertase, C3bBb. This amplification step is tightly controlled by multiple regulators of complement (e.g., CFH, MCP, DAF, and CFI). (C) Once C3 convertase amplification is allowed to proceed, additional C3b is generated, ultimately forming C5 convertase, C3BbC3b. (D) C5 convertase cleaves C5 into C5b, which recruits C6, 7, 8, and 9 to form the membrane attack complex.

the complement system and aHUS, genetic abnormalities identified in familial studies, factors associated with incomplete penetrance, and current methods of diagnosis and treatment.

\section{The Complement System}

The complement system is an essential component of the innate immunity (Figure 1). Its four major steps are: (1) the initiation of the complement cascade; (2) C3 convertase activation and amplification; (3) C5 convertase activation; (4) terminal pathway activation [10]. Initiation of the complement cascade occurs through three pathways: the classical pathway $[11,12]$, the lectin pathway $[13,14]$, and the alternative pathway $[15,16]$. Once activated, C3 convertases are formed (the alternative pathway forms $\mathrm{C} 3 \mathrm{bBb}$, and the classical pathway or the lectin pathway forms C4bC2a), which cleave $\mathrm{C} 3$ to $\mathrm{C} 3 \mathrm{a}$ and $\mathrm{C} 3 \mathrm{~b}$. $\mathrm{C} 3 \mathrm{~b}$ can indiscriminately bind to surfaces of microbes and host cells $[17,18]$. On the surface of microbes or modified host cells, C3b and factor B form more C3 convertases, which produce more C3b. This amplification process exponentially increases the amount of $\mathrm{C} 3 \mathrm{~b}$ and $\mathrm{C} 3$ convertases.

On the surface of intact host cells, in contrast, C3b deposition and C3 convertase amplification are prevented by complement regulators. Regulators distribute in the fluid phase (CFH, CFHR1, CFP, etc.) and on cell surfaces (CR1, MCP, DAF, etc.) to control complement activity through two major mechanisms: decay acceleration activity and cofactor activity [10]. CFH, for example, acts as a cofactor with CFI to cleave $\mathrm{C} 3 \mathrm{~b}$ to an inactive form, $\mathrm{iC} 3 \mathrm{~b}$; has the decay acceleration activity, which promotes the decay of the C3 convertase $[19,20]$ and competes with CFB for binding to $\mathrm{C} 3 \mathrm{~b}$. If $\mathrm{C} 3$ convertase amplification is allowed to proceed unchecked, additional $\mathrm{C} 3 \mathrm{~b}$ binds to $\mathrm{C} 3$ convertases to generate $\mathrm{C} 5$ convertases (C3bBbC3b or C4bC2aC3b) [21]. C5 convertases cleave C5 to C5a and C5b to initiate the terminal pathway and form terminal complement complexes with C6, C7, C8, and C9 to lyse target cells [22, 23].

Mutations of complement genes can either change expression level or disrupt protein function. Figure 2 shows a model of dysregulation of complement regulators inducing aHUS. Mutations in complement genes impair the regulation of $\mathrm{C} 3 \mathrm{~b}$ on host cells, leading to formation of membrane attack complex and host cell damage. Most genetic abnormalities in aHUS patients are found in complement membrane regulators and C3 convertases. Multiple genetic and environmental risk factors are believed necessary to develop disease; however, relatively little is known about how environmental triggers affect homeostasis of complement system in the face of predisposing genetic variants in complement genes [8]. It is also unclear whether genetic variants in complement gene increase susceptibility to typical HUS $[24,25]$.

\section{Genetic Abnormality in Family Cases}

The term "familial aHUS" is used to describe families in which two or more persons develop aHUS at different times without exposure to common triggering infectious agents, or when disease-causing mutations are identified in one of 


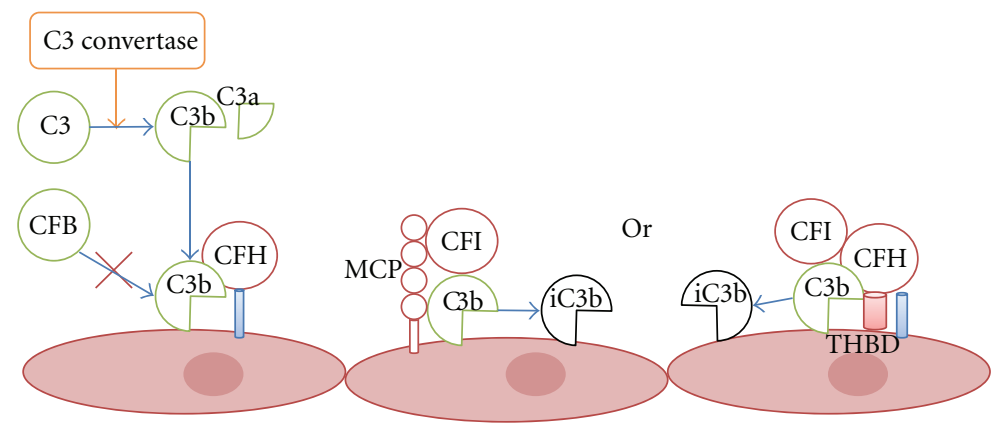

(a)

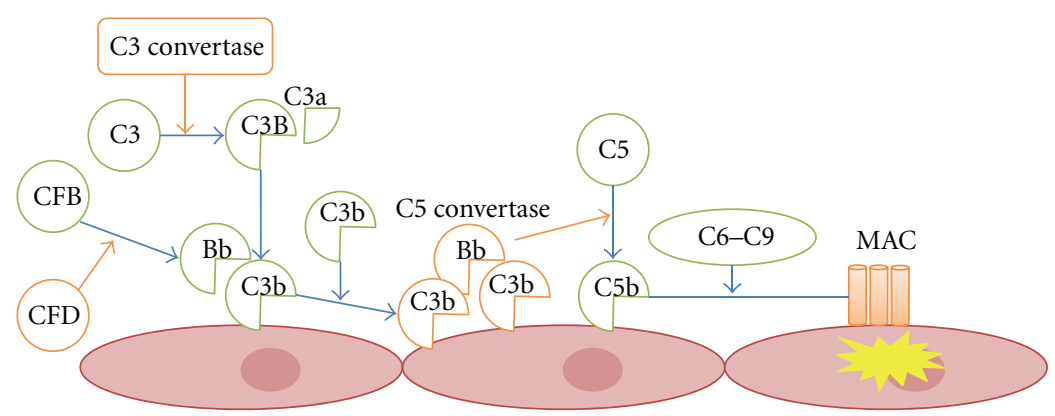

(b)

FIGURE 2: Regulation and dysregulation of complement activity on host cells. C3b is generated by the classical, lectin, or alternative pathways. (a) To protect normal host cells, $\mathrm{C} 3 \mathrm{~b}$ is inactivated by membrane regulators, such as factor $\mathrm{H}(\mathrm{CFH})$ and membrane cofactor protein $(\mathrm{MCP})$. Factor I (CFI) cleaves $\mathrm{C} 3 \mathrm{~b}$ into $\mathrm{iC} 3 \mathrm{~b}$ and other $\mathrm{C} 3$ degradation products with the activity of cofactor regulators. (b) If genetic and/or environmental risk factors reduce the efficiency of membrane complement regulators, $\mathrm{C} 3$ convertase $(\mathrm{C} 3 \mathrm{bBb})$ can accumulate on cell surfaces, creating a C3b amplification loop. Formation of C5 convertase (C3bBbC3b) triggers C5 cleavage into C5b, which interacts with C6, C7, C8, and C9 to generate membrane attack complex (MAC) leading to cell damage.

the genes (discussed next) known to be associated with aHUS irrespective of familial history [1]. Thus, genetic aHUS can be multiplex (two or more affected family members) or simplex (a single occurrence in a family). Since simplex cases develop in patients who do not have a family history of disease, these cases are also referred to as sporadic [1].

The first familial aHUS case was reported in concordant monozygotic twins in 1965 [26]. Since that report, autosomal dominant and recessive familial aHUS has been reported. Familial studies have revealed important genetic factors contributing to aHUS, including mutations in $C F H, C F H R 3$, $M C P, C F I, C F B$, and C3 (Table 1). Most of these mutations impair protein function, causing dysregulation of the complement pathway once it has been activated. Interestingly, some genes implicated in sporadic aHUS, such as THBD [5], have not been associated with familial cases to date. It is reasonable to expect that comprehensive genetic screening of genes in the complement and coagulation pathways will identify variants in additional genes that impact disease penetrance, consistent with aHUS being a complex genetic disease.

3.1. CFH and CFHR Mutations. Complement factor $\mathrm{H}$, encoded by $\mathrm{CFH}$ gene, is an essential inhibitor of $\mathrm{C} 3$ convertase and a central regulator of the complement alternative pathway. It is produced by liver as a soluble protein but can attach to and act on cell surfaces. $\mathrm{CFH}$ protein contains 20 repetitive units of about 60 amino acids named short consensus repeats (SCRs; also known as complement control protein (CCP) repeats or Sushi domains) [27]. N-terminal SCRs regulate binding to $\mathrm{C} 3 \mathrm{~b}$, while C-terminal SCRs facilitate cell-surface binding and regulation. $\mathrm{CFH}$ regulates the complement system through three mechanisms: (1) inhibiting the assembly of C3 convertase by competitive binding to $\mathrm{C} 3 \mathrm{~b},(2)$ accelerating the decay of C3 convertase, and (3) acting as a cofactor in the cleavage and degradation of $\mathrm{C} 3 \mathrm{~b}$ by CFI [27].

$\mathrm{CFH}$ is the most thoroughly studied gene in aHUS. Mutations associated with aHUS were first identified in $\mathrm{CFH}$ by a familial genetic study in 1998 when Warwicker and colleagues conducted linkage analysis in three aHUS families and mapped the aHUS risk region to a $26-\mathrm{cM}$ interval on chr $1 \mathrm{q} 32$ with a lod score of 3.94. The linked region includes the $\mathrm{CFH}$ and $\mathrm{CFHR}$ genes, and in sequencing $\mathrm{CFH}$, a heterozygous c.3716C $>\mathrm{G}$ variant changing an arginine to glycine was found to cosegregate with disease in family 2 . In family 3 , a CFH deletion, c.145_148delAGAA, was found [28].

Numerous missense transversion and transition variants in $\mathrm{CFH}$ have now been associated with aHUS through familial studies [29-34]. Typically, affected patients are heterozygous for these changes, which predominantly occur in the Cterminal SCRs 19 and 20. Since these SCRs are essential for 


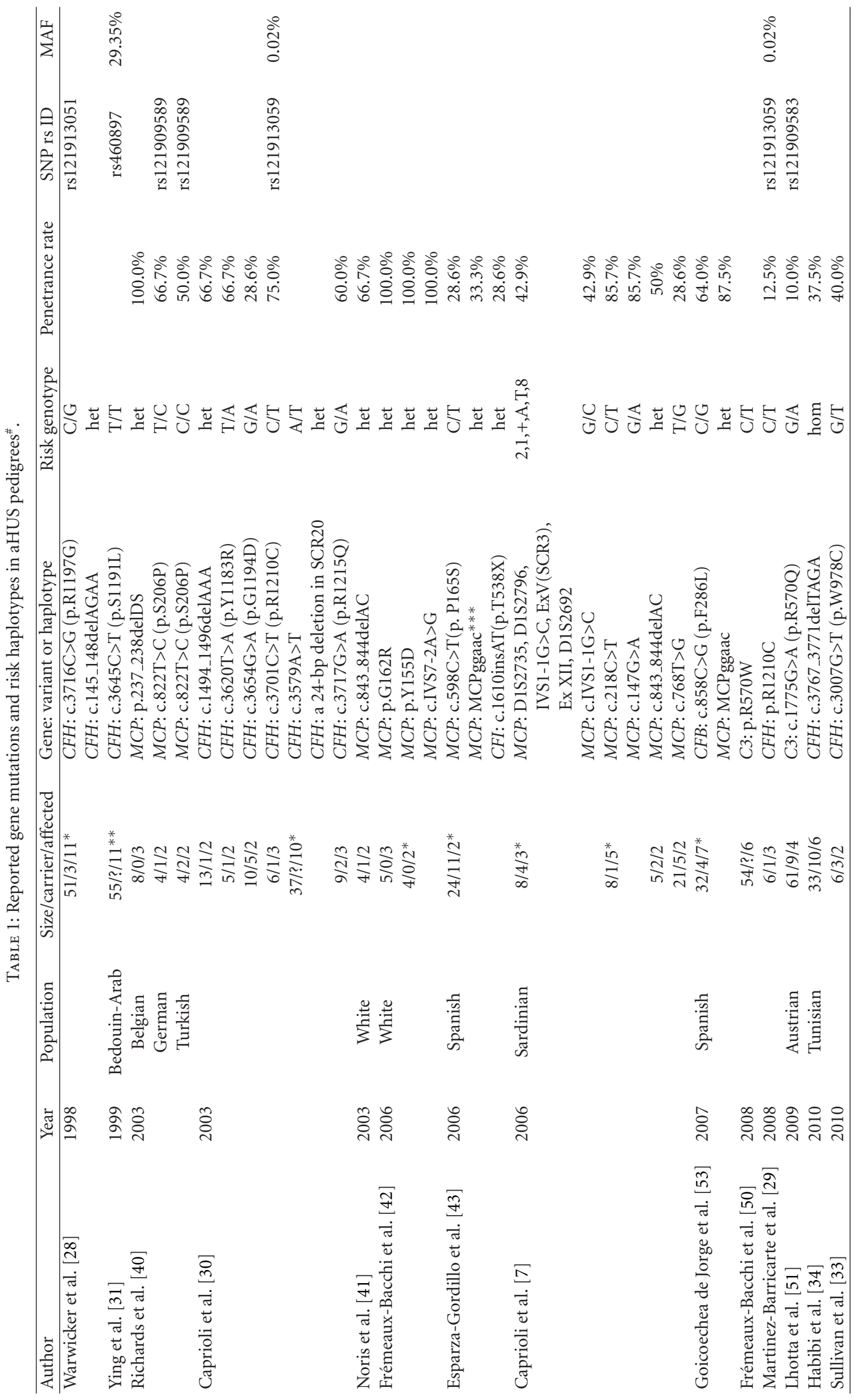




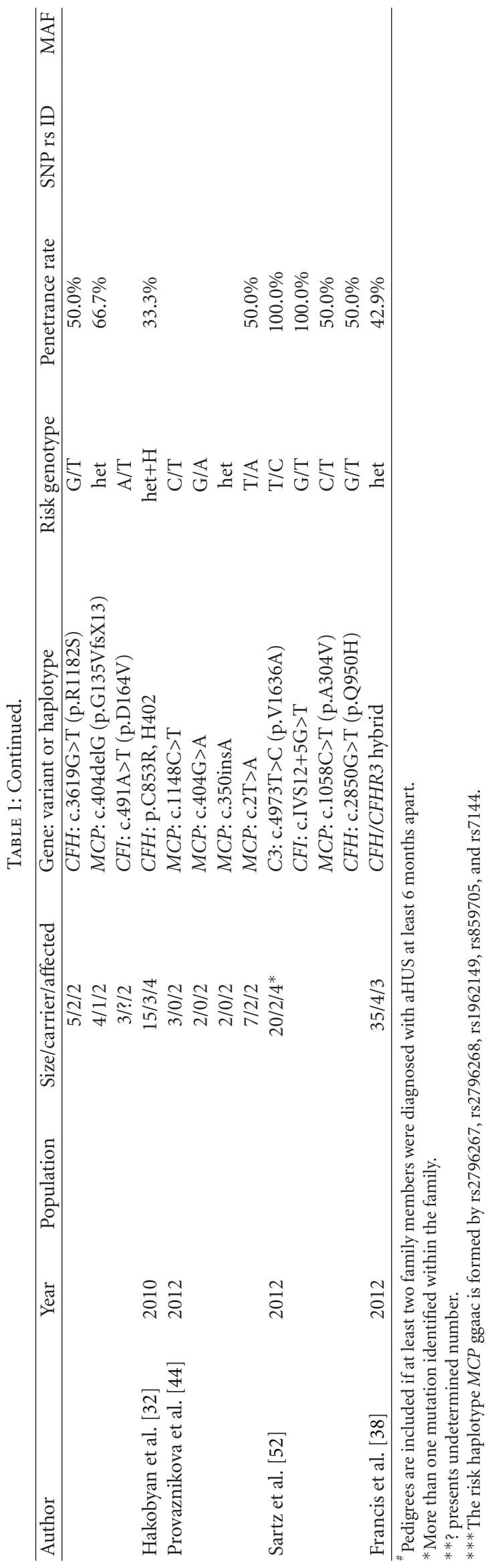


cell-surface attachment, this finding suggests that membrane dysregulation of the complement system is critical to the pathogenesis of aHUS.

Based on available studies, we estimate that penetrance of CFH mutations ranges from $12.5 \%$ to $100 \%$ (Table 1 ). It is remarkable that one $C F H$ rare variant, rs121913059 (c.3701C $>$ T or p.R1210C), has been reported in five families from three familial studies $[29,30,32]$. This variant decreases $\mathrm{CFH}$ binding to $\mathrm{C} 3 \mathrm{~b}$, heparin, and endothelial cells, and leads to a positive sheep erythrocyte hemolytic assay [35]. The Y402H variant (rs1061170, c.1204T $>$ C) of $C F H$, notable for its association with age-related macular degeneration [36], dense deposit disease, and C3 glomerulonephritis [37], has not been associated with aHUS. However, Hakobyan et al. have reported low expression of the CFH-H402 allele in association with other known aHUS variants in two aHUS families, suggesting that in some instances the CFH-H402 allele may contribute to the aHUS phenotype [32].

In addition to $C F H, C F H R 3$ has been linked with familial aHUS. The CFH-related genes (CFHR1, CFHR2, CFHR3, CFHR4, and CFHR5) localize next to CFH and share many of the functional properties of $C F H$. A recent study has reported a hybrid $\mathrm{CFH} / \mathrm{CFHR} 3$ gene caused by a microhomologymediated deletion that is associated with familial aHUS. The transcript product of the hybrid gene contains 24 SCRs with SCRs 1-19 deriving from $C F H$ and SCRs 20-24 deriving from CFHR3. The hybrid protein shows normal fluid-phase activity but loses complement regulation on cell surfaces [38].

3.2. MCP Mutations. MCP (CD46) encodes membrane cofactor protein, which acts as a cofactor for CFI to regulate complement activity by cleaving $\mathrm{C} 3 \mathrm{~b}$ and $\mathrm{C} 4 \mathrm{~b}$ deposited on the surface of host cells. MCP is a transmembrane protein with four N-terminal extracellular Sushi domains, a transmembrane domain, and a C-terminal cytoplasmic tail. Sushi domains 3 and 4 are responsible for complement regulation [39].

MCP is well studied in aHUS. In 2003, Richards et al. [40] first reported mutations of $M C P$ in aHUS families. Two mutations were found in three families - a 6 bp deletion (p.237_238delDS) and c.822T >C (p.S206P, rs121909589). The c.822T $>\mathrm{C}$ mutation causes an amino acid change of serine to proline and leads to a significant reduction of C3b binding. Soon afterwards, Noris et al. reported another aHUS family carrying a $5 \mathrm{bp}$ deletion in MCP gene, which causes a premature stop codon in the fourth Sushi domain [41]. Expression analysis showed around 50\% reduction in MCP as compared to healthy controls. Subsequently, several more MCP mutations have been identified in aHUS families [7, 33, 42-44].

Studies indicate that MCP mutations account for up to $15 \%$ of aHUS patients $[7,33,42]$. Although the majority of MCP mutations are heterozygous $(\sim 75 \%)$, some homozygous or compound heterozygous mutations in MCP have been reported [45]. MCP mutations are defined as (a) type I $(\sim 75 \%)$ if they reduce expression on cell surface and (b) type II $(\sim 25 \%)$ if expression is normal but complement regulatory activity is impaired $[46,47]$.
The MCP ggaac haplotype formed by c. $-652 \mathrm{~A}>\mathrm{G}$ $(\mathrm{rs} 2796267), \quad$ c. $-366 \mathrm{~A}>\mathrm{G} \quad(\mathrm{rs} 2796268), \quad$ c.IVS9-78G $>\mathrm{A}$ (rs1962149), c.IVS12+638G >A (rs859705), and c.4070T >C (rs7144), is associated with aHUS in both sporadic and familial cases $[43,48,49]$. Further studies are needed to determine the functional or expression differences between MCP ggaac and normal haplotypes.

3.3. C3 Mutations. Complement component C3 is the keystone in the complement system. It undergoes spontaneous hydrolysis and is also cleaved by $\mathrm{C} 3$ convertase to $\mathrm{C} 3 \mathrm{~b}$ and $\mathrm{C} 3 \mathrm{a}$. C3b interacts with $\mathrm{CFB}$ to form $\mathrm{C} 3 \mathrm{bB}$, which is then cleaved to $\mathrm{C} 3 \mathrm{bBb}$ by $\mathrm{CFD}$ forming the $\mathrm{C} 3$ convertase. Additional $\mathrm{C} 3 \mathrm{~b}$ leads to the formation of $\mathrm{C} 5$ convertase, which activates the terminal pathway. C3 products are key ligands for multiple complement regulators, including $\mathrm{CFH}$ and MCP. Theoretically, mutations influencing C3 binding ability or other functions could disrupt complement regulation and contribute to the development of aHUS.

Reported C3 mutations are heterozygous and localized on both the beta and alpha chains. In 2008, Frémeaux-Bacchi et al. first reported nine mutations of $C 3$ in 14 patients from 11 families, including a p.R570W mutation in a very large family. Five of the nine identified mutations (p.R570Q, p.R570W, p.A1072V, p.D1093N, and p.Q1139K) reduce ligand binding to MCP, making the mutant convertase resistant to cleavage by CFI thus impairing complement regulation of C3 convertase amplification on cell membranes [50]. Lhotta et al. have also reported a large Austrian family carrying the p.R570Q C3 mutation. In their study, they showed reduced or borderline C3 levels in mutation carriers [51]. Recently, another familial C3 mutation, V1636A, has been identified to cause increased affinity of CFB for C3b [52].

3.4. CFB Mutations. Complement factor $\mathrm{B}$, a key component of $\mathrm{C} 3$ convertase $(\mathrm{C} 3 \mathrm{bBb})$, contains three Sushi domains, a vWFA domain, and a peptidase S1 domain. It is cleaved by $\mathrm{CFD}$ into $\mathrm{Ba}$ and $\mathrm{Bb} . \mathrm{Bb}$ is a serine protease, which binds to $\mathrm{C} 3 \mathrm{~b}$ to generate the $\mathrm{C} 3$ convertase.

In 2007, Goicoechea de Jorge et al. reported a CFB gene mutation in an aHUS family with seven patients [53]. Sequence analysis and functional studies indicated that the missense mutation, c.858C $>$ G (p.F286L), in the vWFA domain, caused more rapid formation and a higher level of C3 convertase. Penetrance was incomplete with seven of 11 mutation carriers developing aHUS. Interestingly, the MCP ggaac risk haplotype was found only in patients and one young carrier who is probably still at risk for disease, suggesting that the effects of the CFB variant are modulated by variants in other complement gene.

3.5. CFI Mutations. Complement factor I is an inhibitory regulator of complement system. It cleaves $\mathrm{C} 3 \mathrm{~b}$ or $\mathrm{C} 4 \mathrm{~b}$ with the presence of cofactors, such as $\mathrm{CFH}$ and MCP, to iC3b, which is cleaved to smaller C3 degradation products. Defects in CFI cause multiple complement-related diseases, including aHUS and CFI deficiency (OMIM: 610984), a disease characterized by recurrent infections and glomerulonephritis in some patients [54]. 
Most CFI mutations have been found in sporadic aHUS cases. These mutations either interrupt cofactor activity or impact the expression level of CFI [55]. In familial aHUS studies, CFI mutations have been reported in three pedigrees (Table 1) [33, 43, 52]. In a Spanish family, a heterozygous $2 \mathrm{bp}$ insertion within the coding region of CFI has been identified to cause a premature stop codon, p.T538X, which reduces plasma levels of CFI by $50 \%$. A missense mutation in $M C P$ (c.598C > T) and the MCP ggaac risk haplotype were also identified in this family, with all patients carrying all three genetic risk factors. Nine unaffected persons carry only one or two genetic risk factors, suggesting that it is the combination of mutations and the risk haplotype that are critical to the development of aHUS [43].

3.6. Combined Mutations and Incomplete Penetrance of Familial aHUS. Multiple familial studies have reported that it is the combination of complement gene mutations that contributes to aHUS [29, 30, 32, 42, 43, 52, 53]. For instance, in a study by Sartz et al., four mutations, one each in $C 3, M C P$, $C F I$, and $C F H$, were found in two patients from a single family [52]. The $C 3$ mutation, p.V1636A, increases the affinity for CFB and C3 convertase; the MCP mutation, p.A304V, increases the activation of the alternative pathway on cell surfaces; and although the functional significance of the CFI mutation (c.IVS12+5) and the CFH mutation (p.Q950H) is unknown, they have been reported in other aHUS cases [52]. The aggregate data suggest that accumulated dysregulation by combined mutations impairs the complement system and leads to disease $[29,30,32,42,43,52,53]$. It is unknown whether other complement factors, such as THBD, CR1, C5$C 9$, and $D A F$, contain risk variants that contribute to the mutation/variant load in aHUS.

Incomplete penetrance is widely observed, with the estimated penetrance of aHUS in mutation carriers being about $50 \sim 60 \%[7,8]$. Within families, affected persons may also show different symptoms and onset ages [56]. These findings strongly suggest that most aHUS-associated genetic variants predispose to rather than cause the disease. However, the genetic picture is incomplete as most studies have focused on only the common complement genes in a disease where rare genetic variants in other complement genes and genes in other pathways are likely to be contributory to the phenotype. Importantly, the effect of common variants is probably marginal as demonstrated by Ermini and colleagues who tested 501 SNPs in 47 complement genes in 220 aHUS patients and 549 controls and found disease associations for only CFH, MCP, and the CFHRs [49]. However, until a comprehensive rare variant screen is completed, it will remain very difficult to calculate disease risk for persons in aHUS families.

\section{Diagnosis and Treatment}

aHUS is clinically characterized by microangiopathic hemolytic anaemia (low hemoglobin, high lactic acid dehydrogenase, undetectable or low haptoglobin, presence of schistocytes in the peripheral blood smear, and negative Coombs test), thrombocytopenia (platelets $<150000 / \mathrm{mm}^{3}$ or a documented rapid decrease), and acute kidney injury (AKI) (hematuria, proteinuria, and/or reduced renal function). However, as a systemic disease, aHUS can affect the endothelia of any organ, and extrarenal manifestations including involvement of the central nervous system, liver, heart, pancreas, and skin, are observed in as many as $20 \%$ of patients $[3,4]$. These additional sites of involvement can blur the distinction between aHUS and other primary thrombotic microangiopathies (TMAs), such as STEC-associated HUS, thrombotic thrombocytopenic purpura (TTP), HELLP syndrome (hemolytic anemia, elevated liver enzymes, and low platelets), and transcyanocobalamin deficiency, or TMAs secondary to malignant hypertension, catastrophic antiphospholipid syndrome, or disseminated intravascular coagulation.

The treatment of aHUS is based on two main strategies: supportive treatment and cause-specific treatment. The former is focused on careful fluid, electrolyte, acid-base, and nutritional management, with the use of blood transfusion, antihypertensive medications, and/or dialysis, as needed. Cause-specific treatment includes plasma therapy provided by either plasma infusion (fresh frozen plasma, 20$40 \mathrm{~mL} / \mathrm{kg} /$ day if the patient is not volume overloaded) or by high volume plasma exchange with fresh frozen plasma ( $150 \%$ of plasma volume daily or every other day until clinical remission). A recent impressive improvement in the management of aHUS has been reported with the use of the anti-C5 monoclonal antibody, Eculizumab, which binds to $\mathrm{C} 5$ thereby preventing activation of the terminal complement cascade. This relatively new (since 2009) treatment is continued until stable clinical remission. Whether life-long or recurrence-specific treatment is necessary and how genetics may or may not impact care of persons on Eculizumab have not been determined.

\section{Transplantation}

Only recently has renal transplantation become the treatment of choice for patients in end stage renal disease on chronic dialysis for aHUS. Until the availability of Eculizumab, transplantation was associated with a $40 \%-80 \%$ risk for disease recurrence [3,57-59]. The notable exception was aHUS patients with $M C P$ mutations since MCP is expressed on the renal endothelia and not in the fluid phase. Eculizumab has become a key resource for preventing recurrence following kidney transplantation and for rescue therapy in case of disease recurrence. Combined liver-kidney transplant with preemptive and perioperative plasma therapy [60], although successfully used in the very recent past, in the Eculizumab era no longer appears to be first-line treatment. Transplantation with a living-related donor is not recommended given our current incomplete understanding of the genetics of aHUS.

\section{Conclusion}

Over the past two decades, studies of familial aHUS have greatly increased our understanding of this disease. The identification of genetic variants in complement genes has 
defined the mechanism whereby complement dysregulation at the cell surface level leads to disease. This understanding has culminated in the use of Eculizumab as first-line therapy in disease treatment, significantly changing the care and prognosis of affected patients. However, even with this bright outlook, major challenges remain to understand the complexity of aHUS at the genetic level. It is possible that a more detailed picture of aHUS can be translated into patient-specific short- and long-term therapy with Eculizumab and/or other anticomplement drugs in the developmental pipeline.

\section{Acknowledgments}

This study was supported in part by the Foundation for Children with Atypical HUS. N. Borsa is supported by the Progetto ALICE ONLUS Associazione per la lotta alla SEU.

\section{References}

[1] M. Noris and G. Remuzzi, "Atypical hemolytic-uremic syndrome," The New England Journal of Medicine, vol. 361, no. 17, pp. 1675-1687, 2009.

[2] A. R. Constantinescu, M. Bitzan, L. S. Weiss et al., "Nonenteropathic hemolytic uremic syndrome: causes and shortterm course," American Journal of Kidney Diseases, vol. 43, no. 6, pp. 976-982, 2004.

[3] M. Noris, J. Caprioli, E. Bresin et al., "Relative role of genetic complement abnormalities in sporadic and familial aHUS and their impact on clinical phenotype," Clinical Journal of the American Society of Nephrology, vol. 5, no. 10, pp. 1844-1859, 2010.

[4] A. L. Sellier-Leclerc, V. Frémeaux-Bacchi, M. A. DragonDurey et al., "Differential impact of complement mutations on clinical characteristics in atypical hemolytic uremic syndrome," Journal of the American Society of Nephrology, vol. 18, no. 8, pp. 2392-2400, 2007.

[5] M. Noris, G. Remuzzi et al., "Atypical hemolytic-uremic syndrome," in GeneReviews, R. A. Pagon, T. D. Bird, C. R. Dolan et al., Eds., Seattle, Wash, USA, 1993.

[6] M. A. Dragon-Durey, S. K. Sethi, A. Bagga et al., "Clinical features of anti-factor $\mathrm{H}$ autoantibody-associated hemolytic uremic syndrome," Journal of the American Society of Nephrology, vol. 21, no. 12, pp. 2180-2187, 2010.

[7] J. Caprioli, M. Noris, S. Brioschi et al., "Genetics of HUS: the impact of $\mathrm{MCP}, \mathrm{CFH}$, and IF mutations on clinical presentation, response to treatment, and outcome," Blood, vol. 108, no. 4, pp. 1267-1279, 2006.

[8] S. R. de Córdoba, "AHUS: a disorder with many risk factors," Blood, vol. 115, no. 2, pp. 158-160, 2010.

[9] J. H. Brown, J. Tellez, V. Wilson et al., "Postpartum aHUS secondary to a genetic abnormality in factor $\mathrm{H}$ acquired through liver transplantation," American Journal of Transplantation, vol. 12, no. 6, pp. 1632-1636, 2012.

[10] P. F. Zipfel and C. Skerka, "Complement regulators and inhibitory proteins," Nature Reviews Immunology, vol. 9, no. 10, pp. 729-740, 2009.

[11] A. W. Dodds, "Which came first, the lectin/classical pathway or the alternative pathway of complement?" Immunobiology, vol. 205, no. 4-5, pp. 340-354, 2002.

[12] R. R. Porter and K. B. M. Reid, "Activation of the complement system by antibody-antigen complexes: the classical pathway," Advances in Protein Chemistry, vol. 33, pp. 1-71, 1979.
[13] M. W. Turner, "The lectin pathway of complement activation," Research in Immunology, vol. 147, no. 2, pp. 110-115, 1996.

[14] M. Matsushita, "The lectin pathway of the complement system," Microbiology and Immunology, vol. 40, no. 12, pp. 887893, 1996.

[15] V. M. Holers, "The spectrum of complement alternative pathway-mediated diseases," Immunological Reviews, vol. 223, no. 1, pp. 300-316, 2008.

[16] M. K. Pangburn and H. J. Muller-Eberhard, "The alternative pathway of complement," Springer Seminars in Immunopathology, vol. 7, no. 2-3, pp. 163-192, 1984.

[17] M. K. Liszewski, C. J. Fang, and J. P. Atkinson, "Inhibiting complement activation on cells at the step of C3 cleavage," Vaccine, vol. 26, supplement 8, pp. I22-I27, 2008.

[18] B. J. C. Janssen and P. Gros, "Conformational complexity of complement component C3," Advances in Experimental Medicine and Biology, vol. 586, pp. 291-312, 2006.

[19] S. J. Perkins, R. Nan, A. I. Okemefuna, K. Li, S. Khan, and A. Miller, "Multiple interactions of complement factor h with its ligands in solution: a progress report," Advances in Experimental Medicine and Biology, vol. 703, pp. 25-47, 2010.

[20] C. Skerka and P. F. Zipfel, "Complement factor H related proteins in immune diseases," Vaccine, vol. 26, supplement 8, pp. I9-I14, 2008.

[21] M. K. Pangburn and N. Rawal, "Structure and function of complement C5 convertase enzymes," Biochemical Society Transactions, vol. 30, no. 6, pp. 1006-1010, 2002.

[22] B. P. Morgan, "Regulation of the complement membrane attack pathway," Critical Reviews in Immunology, vol. 19, no. 3, pp. 173-198, 1999.

[23] H. J. Muller-Eberhard, "The membrane attack complex of complement," Annual Review of Immunology, vol. 4, pp. 503528, 1986.

[24] T. N. Petruzziello-Pellegrini and P. A. Marsden, "Shiga toxinassociated hemolytic uremic syndrome: advances in pathogenesis and therapeutics," Current Opinion in Nephrology and Hypertension, vol. 21, no. 4, pp. 433-440, 2012.

[25] K. Poolpol, B. Gadner, S. Neururer et al., "Do complement factor $\mathrm{H} 402 \mathrm{Y}$ and $\mathrm{C7} \mathrm{M}$ allotypes predispose to (typical) haemolytic uraemic syndrome?" International Journal of Immunogenetics, vol. 38, no. 5, pp. 383-387, 2011.

[26] S. Campbell and I. J. Carré, "Fatal haemolytic uraemic syndrome and idiopathic hyperlipaemia in monozygotic twins," Archives of Disease in Childhood, vol. 40, no. 214, pp. 654-658, 1965.

[27] C. J. F. Boon, N. C. van de Kar, B. J. Klevering et al., "The spectrum of phenotypes caused by variants in the CFH gene," Molecular Immunology, vol. 46, no. 8-9, pp. 1573-1594, 2009.

[28] P. Warwicker, T. H. J. Goodship, R. L. Donne et al., "Genetic studies into inherited and sporadic hemolytic uremic syndrome," Kidney International, vol. 53, no. 4, pp. 836-844, 1998.

[29] R. Martinez-Barricarte, G. Pianetti, R. Gautard et al., "The complement factor H R1210C mutation is associated with atypical hemolytic uremic syndrome," Journal of the American Society of Nephrology, vol. 19, no. 3, pp. 639-646, 2008.

[30] J. Caprioli, F. Castelletti, S. Bucchioni et al., "Complement factor $\mathrm{H}$ mutations and gene polymorphisms in haemolytic uraemic syndrome: the C-257T, the A2089G and the G2881T polymorphisms are strongly associated with the disease," Human Molecular Genetics, vol. 12, no. 24, pp. 3385-3395, 2003.

[31] L. Ying, Y. Katz, M. Schlesinger et al., "Complement factor $\mathrm{H}$ gene mutation associated with autosomal recessive atypical hemolytic uremic syndrome," American Journal of Human Genetics, vol. 65, no. 6, pp. 1538-1546, 1999. 
[32] S. Hakobyan, A. Tortajada, C. L. Harris, S. R. De Córdoba, and B. P. Morgan, "Variant-specific quantification of factor $\mathrm{H}$ in plasma identifies null alleles associated with atypical hemolytic uremic syndrome," Kidney International, vol. 78, no. 8, pp. 782-788, 2010.

[33] M. Sullivan, Z. Erlic, M. M. Hoffmann et al., "Epidemiological approach to identifying genetic predispositions for atypical hemolytic uremic syndrome," Annals of Human Genetics, vol. 74, no. 1, pp. 17-26, 2010.

[34] I. Habibi, I. Sfar, W. Ben Alaya et al., "Atypical hemolytic uremic syndrome and mutation analysis of factor $\mathrm{H}$ gene in two Tunisian families," International Journal of Nephrology and Renovascular Disease, vol. 3, pp. 85-92, 2010.

[35] L. T. Roumenina, C. Loirat, M. A. Dragon-Durey, L. Halbwachs-Mecarelli, C. Sautes-Fridman, and V. FrémeauxBacchi, "Alternative complement pathway assessment in patients with atypical HUS," Journal of Immunological Methods, vol. 365, no. 1-2, pp. 8-26, 2011.

[36] R. Sofat, J. P. Casas, A. R. Webster et al., "Complement factor $\mathrm{H}$ genetic variant and age-related macular degeneration: effect size, modifiers and relationship to disease subtype," International Journal of Epidemiology, vol. 41, no. 1, pp. 250-262, 2012.

[37] A. Servais, L. H. Noël, L. T. Roumenina et al., "Acquired and genetic complement abnormalities play a critical role in dense deposit disease and other C3 glomerulopathies," Kidney International, vol. 82, no. 4, pp. 454-464, 2012.

[38] N. J. Francis, B. McNicholas, A. Awan et al., "A novel hybrid CFH/CFHR3 gene generated by a microhomology-mediated deletion in familial atypical hemolytic uremic syndrome," Blood, vol. 119, no. 2, pp. 591-601, 2012.

[39] J. Cardone, G. Le Friec, and C. Kemper, "CD46 in innate and adaptive immunity: an update," Clinical and Experimental Immunology, vol. 164, no. 3, pp. 301-311, 2011.

[40] A. Richards, E. J. Kemp, M. K. Liszewski et al., "Mutations in human complement regulator, membrane cofactor protein (CD46), predispose to development of familial hemolytic uremic syndrome," Proceedings of the National Academy of Sciences of the United States of America, vol. 100, no. 22, pp. 12966-12971, 2003.

[41] M. Noris, S. Brioschi, J. Caprioli et al., "Familial haemolytic uraemic syndrome and an MCP mutation," The Lancet, vol. 362, no. 9395, pp. 1542-1547, 2003.

[42] V. Frémeaux-Bacchi, E. A. Moulton, D. Kavanagh et al., "Genetic and functional analyses of membrane cofactor protein (CD46) mutations in atypical hemolytic uremic syndrome," Journal of the American Society of Nephrology, vol. 17, no. 7, pp. 2017-2025, 2006.

[43] J. Esparza-Gordillo, E. G. D. Jorge, C. A. Garrido et al., "Insights into hemolytic uremic syndrome: segregation of three independent predisposition factors in a large, multiple affected pedigree," Molecular Immunology, vol. 43, no. 11, pp. 1769-1775, 2006

[44] D. Provaznikova, S. Rittich, M. Malina et al., "Manifestation of atypical hemolytic uremic syndrome caused by novel mutations in MCP," Pediatric Nephrology, vol. 27, no. 1, pp. 73-81, 2012.

[45] M. K. Liszewski, C. Kemper, J. D. Price, and J. P. Atkinson, "Emerging roles and new functions of CD46," Springer Seminars in Immunopathology, vol. 27, no. 3, pp. 345-358, 2005.

[46] A. Richards, M. Kathryn Liszewski, D. Kavanagh et al., "Implications of the initial mutations in membrane cofactor protein (MCP; CD46) leading to atypical hemolytic uremic syndrome," Molecular Immunology, vol. 44, no. 1-3, pp. 111-122, 2007.
[47] R. E. Saunders, C. Abarrategui-Garrido, V. Frémeaux-Bacchi et al., "The interactive factor $\mathrm{H}$-atypical hemolytic uremic syndrome mutation database and website: update and integration of membrane cofactor protein and factor I mutations with structural models," Human Mutation, vol. 28, no. 3, pp. 222234, 2007.

[48] J. Esparza-Gordillo, E. Goicoechea de Jorge, A. Buil et al., "Predisposition to atypical hemolytic uremic syndrome involves the concurrence of different susceptibility alleles in the regulators of complement activation gene cluster in 1q32," Human Molecular Genetics, vol. 14, no. 5, pp. 703-712, 2005.

[49] L. Ermini, T. H. Goodship, L. Strain et al., "Common genetic variants in complement genes other than $\mathrm{CFH}, \mathrm{CD} 46$ and the CFHRs are not associated with aHUS," Molecular Immunology, vol. 49, no. 4, pp. 640-648, 2012.

[50] V. Frémeaux-Bacchi, E. C. Miller, M. K. Liszewski et al., "Mutations in complement C3 predispose to development of atypical hemolytic uremic syndrome," Blood, vol. 112, no. 13, pp. 4948-4952, 2008.

[51] D. K. Lhotta, A. R. Janecke, J. Scheiring et al., "A large family with a gain-of-function mutation of complement C3 predisposing to atypical hemolytic uremic syndrome, microhematuria, hypertension and chronic renal failure," Clinical Journal of the American Society of Nephrology, vol. 4, no. 8, pp. 13561362, 2009.

[52] L. Sartz, A. I. Olin, A. C. Kristoffersson et al., "A novel C3 mutation causing increased formation of the $\mathrm{C} 3$ convertase in familial atypical hemolytic uremic syndrome," Journal of Immunology, vol. 188, no. 4, pp. 2030-2037, 2012.

[53] E. Goicoechea de Jorge, C. L. Harris, J. Esparza-Gordillo et al., "Gain-of-function mutations in complement factor B are associated with atypical hemolytic uremic syndrome," Proceedings of the National Academy of Sciences of the United States of America, vol. 104, no. 1, pp. 240-245, 2007.

[54] C. A. Alper, F. S. Rosen, and P. J. Lachmann, "Inactivator of the third component of complement as an inhibitor in the properdin pathway," Proceedings of the National Academy of Sciences of the United States of America, vol. 69, no. 10, pp. 29102913, 1972.

[55] S. C. Nilsson, R. B. Sim, S. M. Lea et al., "Complement factor I in health and disease," Molecular Immunology, vol. 48, no. 14, pp. 1611-1620, 2011.

[56] M. Sullivan, L. A. Rybicki, A. Winter et al., "Age-related penetrance of hereditary atypical hemolytic uremic syndrome," Annals of Human Genetics, vol. 75, no. 6, pp. 639-647, 2011.

[57] D. Westra, J. F. Wetzels, E. B. Volokhina et al., "A new era in the diagnosis and treatment of atypical haemolytic uraemic syndrome," The Netherlands Journal of Medicine, vol. 70, no. 3, pp. 121-129, 2012.

[58] C. Loirat and V. Frémeaux-Bacchi, "Atypical hemolytic uremic syndrome," Orphanet Journal of Rare Diseases, vol. 6, p. 60, 2011.

[59] J. Zuber, M. Le Quintrec, R. Sberro-Soussan, C. Loirat, V. Frémeaux-Bacchi, and C. Legendre, "New insights into postrenal transplant hemolytic uremic syndrome," Nature Reviews Nephrology, vol. 7, no. 1, pp. 23-35, 2011.

[60] J. M. Saland, P. Ruggenenti, G. Remuzzi, and Consensus Study Group, "Liver-kidney transplantation to cure atypical hemolytic uremic syndrome," Journal of the American Society of Nephrology, vol. 20, no. 5, pp. 940-949, 2009. 


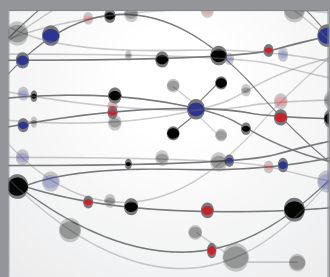

The Scientific World Journal
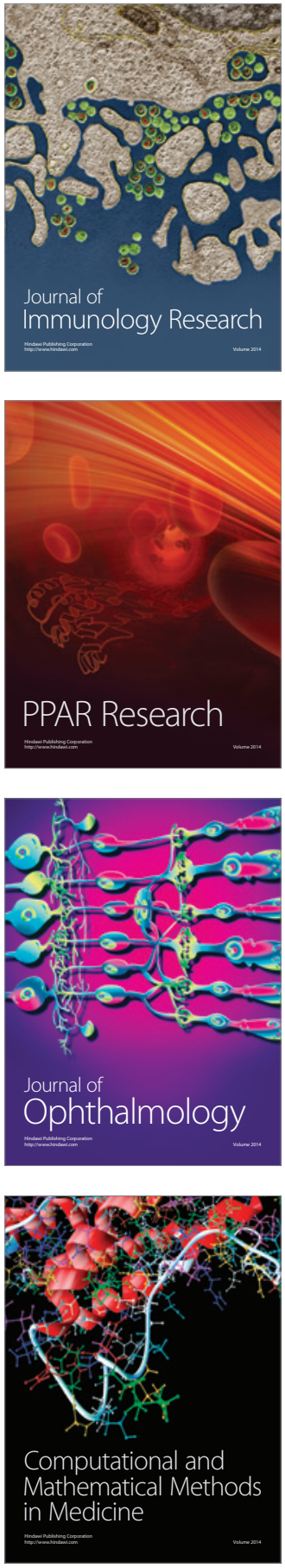

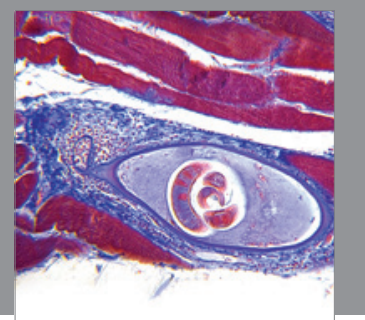

Gastroenterology

Research and Practice
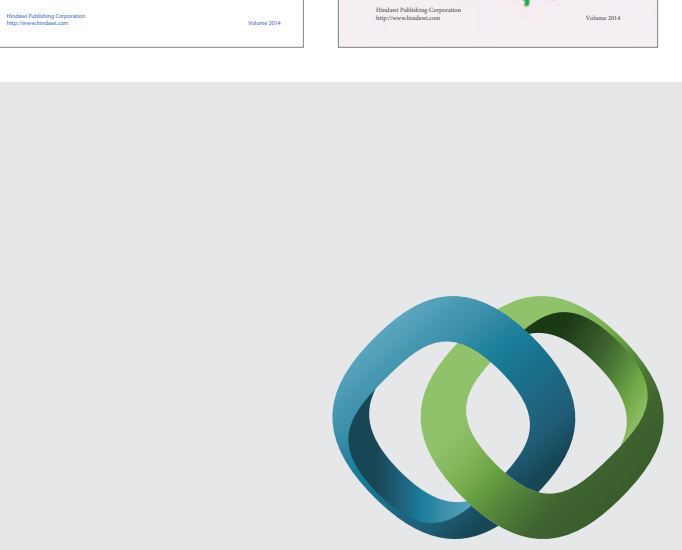

\section{Hindawi}

Submit your manuscripts at

http://www.hindawi.com
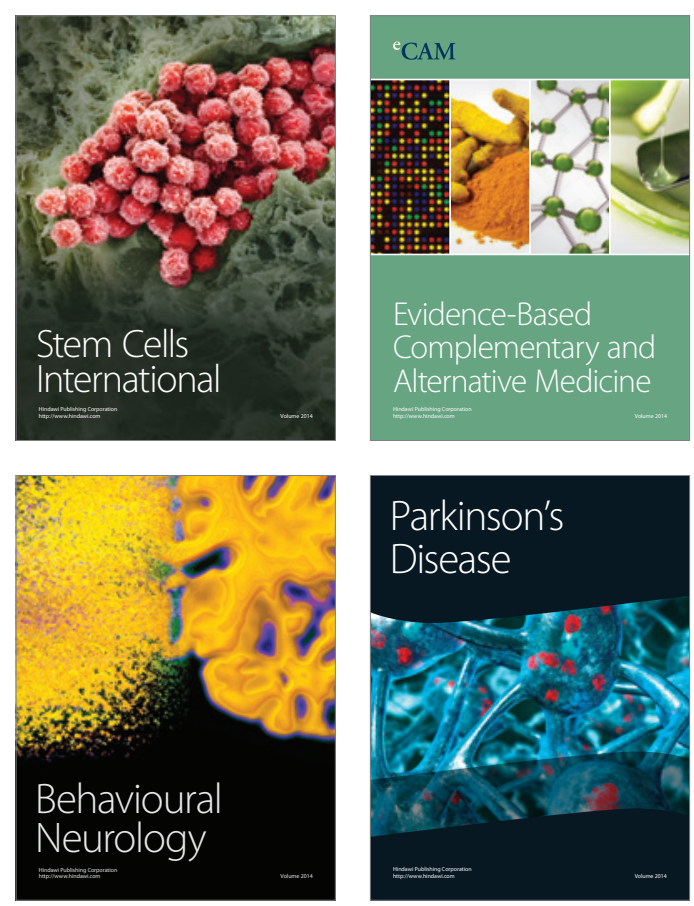

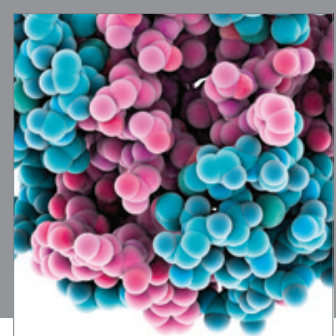

Journal of
Diabetes Research

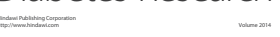

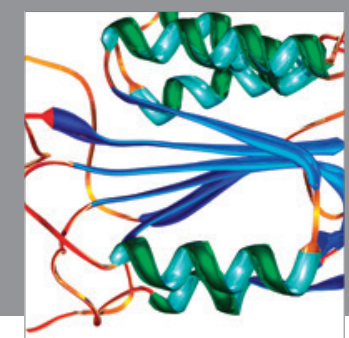

Disease Markers
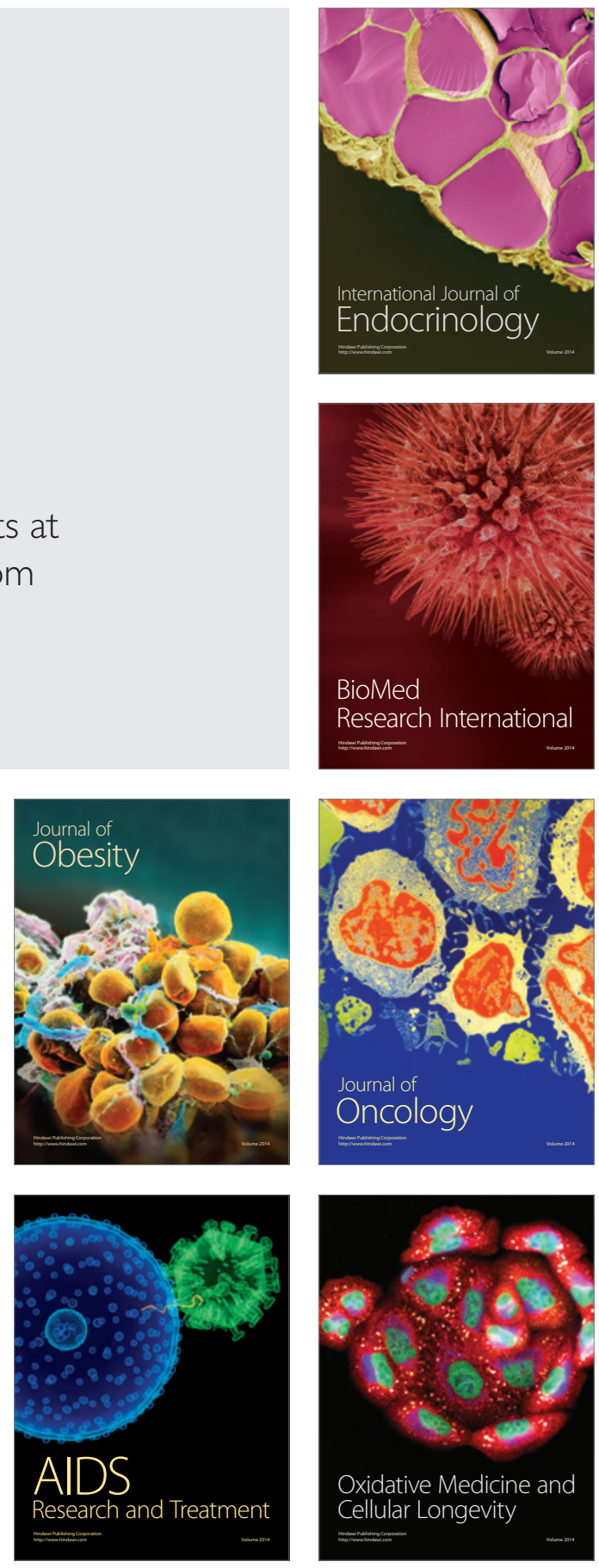